This item was submitted to Loughborough's Institutional Repository (https://dspace.lboro.ac.uk/) by the author and is made available under the following Creative Commons Licence conditions.

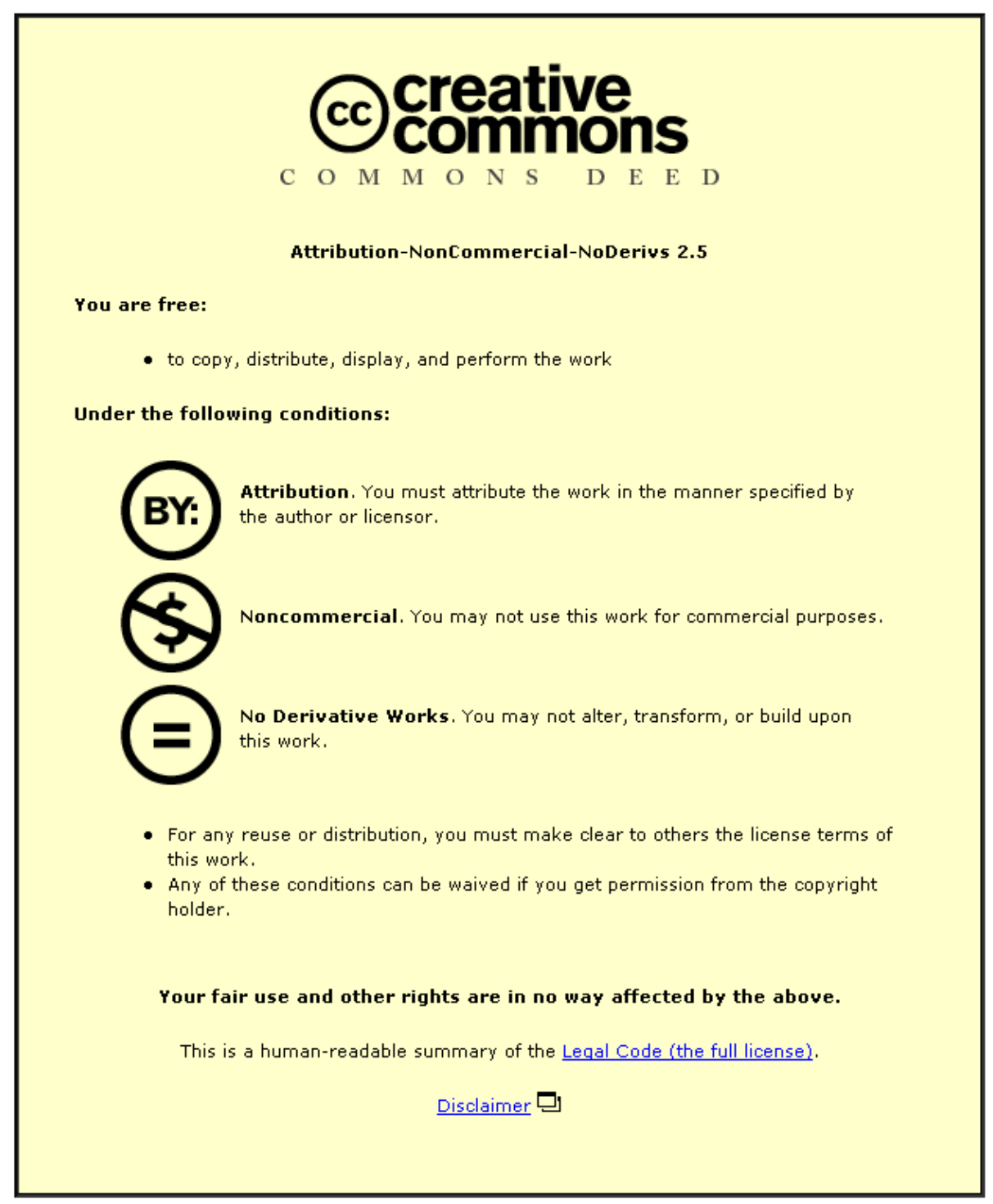

For the full text of this licence, please go to: http://creativecommons.org/licenses/by-nc-nd/2.5/ 


\title{
Adaptive Signal Processing Techniques For Clutter Removal In Radar-based Navigation Systems
}

\author{
Yanfeng Liang ${ }^{1,2}$ Wenwu Wang ${ }^{3}$ Jonathon Chambers $^{2}$ \\ 1. College of Automation, Harbin Engineering University, China \\ 2. Electronic and Electrical Engineering Department, Loughborough University, UK \\ 3. Department of Electronic Engineering, University of Surrey, UK \\ Email: peakbeam@yahoo.com.cn,w.wang@surrey.ac.uk,eljac@lboro.ac.uk
}

\begin{abstract}
The problem of background clutter remains as a major challenge in radar-based navigation, particularly due to its time-varying statistical properties. Adaptive solutions for clutter removal are therefore sought which meet the demanding convergence and accuracy requirements of the navigation application. In this paper, a new structure which combines blind source separation (BSS) and adaptive interference cancellation (AIC) is proposed to solve the problem more accurately without prior statistical knowledge of the sea clutter. The new algorithms are confirmed to outperform previously proposed adaptive schemes for such processing through simulation studies.
\end{abstract}

Index Terms - adaptive signal processing, sea clutter removal, blind source separation, adaptive interference cancellation

\section{INTRODUCTION}

In ship navigation systems, there are many pieces of navigation equipment such as GPS (Global Positioning System), ECDIS (Electronic Chart Display and Information System), gyroscopes, sonar and radar. Navigation radar is an important element of such equipment for the following reasons. First of all, radar can detect other ships thereby avoiding collisions. Secondly, radar can also detect the coastline and islands to avoid tragic events. Although ECDIS can provide such information, ECDIS combined with radar detection can improve the accuracy of the whole navigation system. Thirdly, radar can also have the ability to examine the GPS accuracy. Thus, radar plays an important role in ship navigation systems. However, there is always a problem for radar in ship navigation systems, the sea clutter. The sea clutter disturbs the detection ability of radar. It makes the real targets hard to recognize. Therefore, methodology for removing the clutter from a radar signal is a very important and practical issue.

A traditional method to detect the presence of a radar return is based on the CFAR method (Constant False Alarm Rate)[1]. Radar detection involves the comparison between a threshold and the radar signal. In order to attain a constant false alarm rate, the CFAR method is introduced to get the adaptive threshold to reflect the local clutter situation. There are many CFAR related methods, such as cell-averaging CFAR (CA-CFAR), greatest of CFAR (GO-CFAR), smallest of CFAR (SO-CFAR) and order statistic CFAR (OS-CFAR)[2]. CA-CFAR method uses the average of the chosen cell to calculate the adaptive threshold. The GO-CFAR method uses the greatest element of the chosen cell to calculate the threshold, while the SOCFAR uses the smallest one. OS-CFAR is an order statistical method which is introduced from image processing. But there is a drawback for these methods, the prior knowledge (the density distribution) describing the sea clutter is needed. It is well known that sea clutter is very hard to describe accurately, which makes the prior assumptions inaccurate. As a result, the effect of removing clutter is not well understood. BSS[3] and AIC[4] have the advantage that no major prior knowledge is needed. So, the sea clutter removal based on BSS and AIC can successfully avoid the problem that sea clutter is too complex to describe. In this paper, first of all, the radar signal is modelled. Then two types of traditional adaptive interference canceler structures are introduced, and the drawbacks of the traditional methods are also indicated. After these analyses, a new method based on BSS and AIC is proposed to solve the radar sea clutter removal problem. This new method does not depend on the sea clutter assumptions, and can provide more stable and more accurate performance. Finally, the experiments are provided to confirm the advantages of this new structure.

\section{Measurement Model}

\section{A. Radar Signal}

A received radar signal typically contains two parts: the target and sea clutter. It is well known that sea clutter is difficult to describe fully statistically. Mostly, there are three types of model to describe sea clutter. They are Rayleigh, Weibull and Log-normal distributions[5]. Generally, the Rayleigh model tends to underestimate the dynamic range of the real clutter distribution while the Log-normal model tends to overestimate the dynamic range. Compared with the other two models, the Weibull distribution can describe the sea clutter more accurately. The Weibull clutter model offers the potential to represent accurately the real clutter distribution over a much wider range of conditions than either the Log-normal or Rayleigh model. By appropriately adjusting its parameters, the Weibull distribution can be made to approach either the Rayleigh (a member of the Weibull family) or log-normal distribution. So, the Weibull distribution is adopted to establish the clutter model in this paper. The Weibull probability density function is written as follows:

$$
p(x)=\frac{c}{b}\left(\frac{x}{b}\right)^{c-1} \exp \left[-\left(\frac{x}{b}\right)^{c}\right] \text { for } x, b, c>0
$$


where $x$ is the amplitude of the return signals, and $b$ is a scale parameter and $\mathrm{c}$ is a shape parameter. For $c=2.0$, the Weibull distribution is identical to the Rayleigh distribution.

When the radar is working, firstly, it emits a series of signals; when these signals meet the target, a reflected signal is generated which can be detected by the radar receiver. So, if there was no sea clutter, the received signal would only have values in certain time intervals, while the rest of the received signal should be zero. In practice, clutter will be present so we must examine schemes for its removal.

\section{B. Traditional Adaptive Interference Canceller Structure}

Traditionally, there are two types of adaptive structures to cancel interference, Fig.1 and Fig.2[4]. The first uses two sensors, with one been used for receiving signal plus interference and the other for detecting correlated interference. A signal is assumed to be transmitted over a channel to a sensor that receives the target signal and uncorrelated interference. The combined signal, forms the primary input to the canceller. A second sensor receives an interference which is uncorrelated with the target signal but correlated with the interference. In this type of system, the reference input is processed by an adaptive filter, and the output of the adaptive filter can be a close replica of the interference. This output is subtracted from the primary input to produce the output of the whole system, namely the target signal without interference. The other type of AIC only uses one sensor to receive signals. A fixed delay of the input signal is taken as the reference input. We must make sure that the delay is of sufficient length so that the broadband signal components in the reference input become de-correlated from those in the primary input, otherwise signal leakage to the reference input degrades performance. For the first type of AIC, the problem is that it is hard to find a method to detect the interferences only, especially for radar. As for the second type, the drawback is that the performance is generally not as good as the first type with two sensors, as it is difficult to select the decorrelation delay.

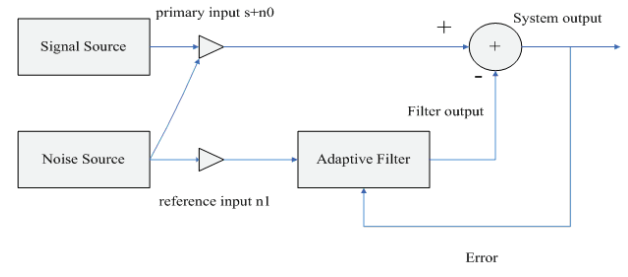

Fig. 1. Two sensor type of AIC processing structure

\section{A New Processing Structure}

In this paper, a new processing structure is therefore proposed to solve the clutter removal problem. In Fig 3, the new structure combines BSS and the first type of AIC. BSS is used to separate the target and sea clutter. BSS can not be used alone to solve this problem, because there are two ambiguities for

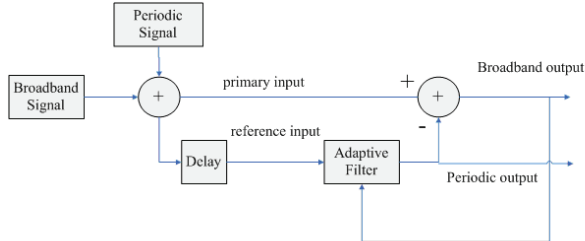

Fig. 2. Single sensor type of AIC processing structure

BSS. One of them is that the independent components can only be estimated up to a nonzero multiplicative constant, since any constant multiplying the sources can be cancelled out by dividing the corresponding column of the mixing matrix by the same constant. This ambiguity leads to the problem that the separated target is sometimes very small, which makes it hard to be recognized. Thus, only using BSS can not achieve the ideal result. However, BSS is still potentially very useful. The separated sea clutter is highly correlated with the real sea clutter. Thus we can use the separated sea clutter signal as the reference signal for the first type of AIC structure.

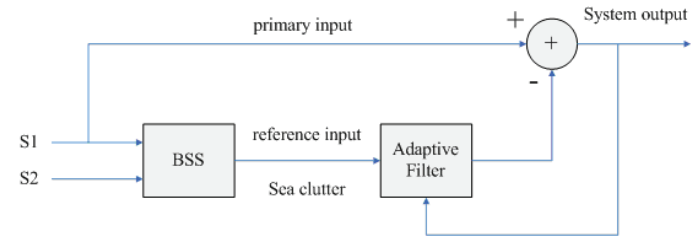

Fig. 3. Combined BSS and AIC processing structure for adaptive noise cancellation

\section{Proposed Adaptive Algorithms For NeW SCHEME}

The new processing structure contains two parts, the BSS and AIC. In the BSS part, the FastICA algorithm[6] is chosen for its good performance. And in the AIC part, the RLS (Recursive Least Square)algorithm[7] is chosen for its fast convergence.

\section{A. FastICA using Negentropy}

Independent component analysis (ICA) is the main method for the BSS problem. The principle of ICA is maximizing non-Gaussianity. Kurtosis and negentropy are used to measure the non-Gaussianity. In this paper, negentropy is used to measure the non-Gaussianity, because it is more accurately than kurtosis[6].

Negentropy is based on the information-theoretic quantity of differential entropy. Entropy is the basic concept of information theory. The entropy $H$ of a random vector $\mathbf{y}$ 
with density $p_{y}(\eta)$ is defined as

$$
H(\mathbf{y})=-\int p_{\mathbf{y}}(\eta) \log \left(p_{\mathbf{y}}(\eta)\right) \mathbf{d} \eta
$$

A fundamental result of information theory is that a Gaussian variable has the largest entropy among all random variables of equal variance. This means that entropy can be used as a measure of non-Gaussianity.

To obtain a measure of non-Gaussianity that is zero for a Gaussian variable and always nonnegative, the negentropy is introduced.

$$
J(\mathbf{y})=H\left(\mathbf{y}_{\text {gauss }}\right)-H(\mathbf{y})
$$

The FastICA algorithm using negentropy involves[6]:

(1). Center the data $\mathbf{s}=\left[\mathbf{s}_{1} ; \mathbf{s}_{2}\right]$ to make its mean zero.

(2). Whiten the data to give $\mathbf{z}$.

(3). Choose $m$, the number of independent components to estimate.

(4). Choose initial values for the $\mathbf{w}_{i}, i=1, \cdots, m$, each of unit norm. Orthogonalize the matrix $\mathbf{W}, \mathbf{W}=$ $\left(\mathbf{w}_{1}, \cdots, \mathbf{w}_{m}\right)^{T}$

(5). Let $E\left\{\mathbf{z} g\left(\mathbf{w}_{i}^{T} \mathbf{z}\right)\right\}-E\left\{g^{\prime}\left(\mathbf{w}_{i}^{T} \mathbf{z}\right)\right\} \mathbf{w} \rightarrow \mathbf{w}$, for every $i=1, \cdots, m$, where $g=\tanh ()$ and $g^{\prime}=1-\tanh ^{2}()$.

(6). Do a symmetric orthogonalization of the matrix $\mathbf{W}$.

(7). If not converged, return to step (5).

\section{B. AIC based on RLS}

After the BSS, the estimated sea clutter and the estimated target can be attained. The problem is the value of the estimated target can be too small to detect. However, it is found that the estimated clutter fits the real clutter very well, see Fig.6. Now, the estimated sea clutter can be used as the reference input of the first type AIC model. The RLS algorithm is chosen because the target signal is not a statistically stationary signal. When the target appears, RLS can converge fast to track the target.

Derivation of the RLS algorithm[7]: Start from the regularized Newton's recursion

$$
\mathbf{w}_{i}=\mathbf{w}_{i-1}+\mu(i)\left[\epsilon(i) \mathbf{I}+\mathbf{R}_{\mathbf{u}}\right]^{-1}\left[\mathbf{R}_{\mathbf{d u}}-\mathbf{R}_{\mathbf{u}} \mathbf{w}_{i-1}\right]
$$

Replace $\left(\mathbf{R}_{\mathbf{d u}}-\mathbf{R}_{\mathbf{u}} \mathbf{w}_{i-1}\right)$ by the instantaneous approximation $\mathbf{u}_{i}^{*}\left[\mathbf{d}(i)-\mathbf{u}_{i} \mathbf{w}_{i-1}\right]$. Then replace $\mathbf{R}_{\mathbf{u}}$ by an estimate based on the exponentially weighted sample average as follow:

$$
\mathbf{R}_{\mathbf{u}}=\frac{1}{i+1} \sum_{j=0}^{i} \lambda^{i-j} \mathbf{u}_{j}^{*} \mathbf{u}_{j} \text { where } 0 \ll \lambda \leq 1
$$

Make $\mu(i)=1 /(i+1)$ and $\epsilon(i)=\lambda^{i+1} \epsilon /(i+1)$, we have

$$
\begin{array}{r}
\mathbf{w}_{i}=\mathbf{w}_{i-1}+\left[\lambda^{i+1} \epsilon(i) \mathbf{I}+\sum_{j=0}^{i} \lambda^{i-j} \mathbf{u}_{j}^{*} \mathbf{u}_{j}\right]^{-1} \mathbf{u}_{i}^{*}[\mathbf{d}(i)- \\
\left.\mathbf{u}_{i} \mathbf{w}_{i-1}\right]
\end{array}
$$

Let $\boldsymbol{\Phi}_{i}=\left(\lambda^{i+1} \epsilon(i) \mathbf{I}+\sum_{j=0}^{i} \lambda^{i-j} \mathbf{u}_{j}^{*} \mathbf{u}_{j}\right)$
$\boldsymbol{\Phi}_{i} \quad$ satisfies $\boldsymbol{\Phi}_{i}=\lambda \boldsymbol{\Phi}_{i-1}+\mathbf{u}_{i}^{*} \mathbf{u}_{i}$

Make $\mathbf{P}_{i}=\boldsymbol{\Phi}_{i}^{-1}$

$$
\left.\mathbf{P}_{i}=\lambda^{-1} \mathbf{P}_{i-1}-\frac{\lambda^{-1} \mathbf{P}_{i-1} \mathbf{u}_{i}^{*} \mathbf{u}_{i} \mathbf{P}_{i-1}}{1+\lambda^{-1} \mathbf{u}_{i} \mathbf{P}_{i-1} \mathbf{u}_{i}^{*}}\right]
$$

The RLS algorithm is convergent in the mean and mean square if the filter input and the desired response are jointly stationary ergodic processes.Compared with the LMS algorithm, the convergence performance of RLS is significantly superior to that of LMS. The RLS algorithm converges in the mean square in about $2 M$ iterations, where $M$ is the number of taps in the filter. While the LMS algorithm needs about $20 M$ to converge. And the rate of the convergence of the RLS algorithm is essentially insensitive to variations in the eigenvalue spread of the correlation matrix of the input vector. The performance of the RLS algorithm can also be beneficial for duration limited non-stationary signals and on this basis we employ the RLS algorithm in our work. Moreover,In theory, the RLS algorithm produces zero excess mean-squared error in a stationary environment[8]. And therefore it is suitable for practical situations where the clutter might be coloured.

\section{EXPERIMENT}

In this experiment, the target signal is a non-stationary (since it is only present in certain time intervals) sinusoid signal $\mathbf{x}_{1}$ with normalized frequency $p i / 5$ and with 10 samples per period. And the sea clutter signal $\mathbf{x}_{2}$ obeys to Weibull distribution, Fig.4. The two radar received signals $\mathbf{s}_{1}$, $\mathbf{s}_{2}$ are :

$$
\begin{aligned}
& \mathbf{s}_{1}=\alpha_{1} \mathbf{x}_{1}+\beta_{1} \mathbf{x}_{2} \\
& \mathbf{s}_{2}=\alpha_{2} \mathbf{x}_{1}+\beta_{2} \mathbf{x}_{2}
\end{aligned}
$$

In this experiment, $\alpha_{1}=0.3, \beta_{1}=0.8, \alpha_{2}=0.4, \beta_{2}=0.7$. The received signals in Fig.5 show that the sea clutter submerges the target. It is hard to detect the target reliably in the sea clutter.
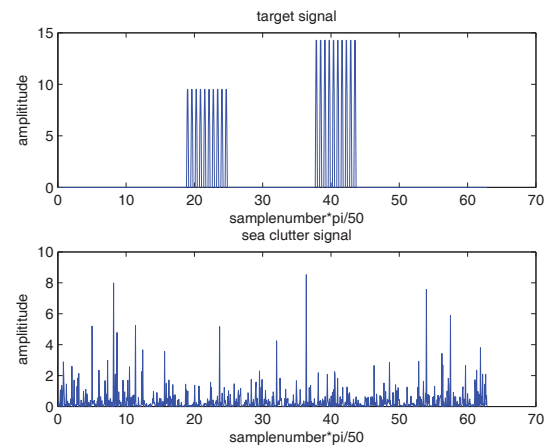

Fig. 4. Target signal and sea clutter signals

The BSS separated target signal can be too small to be detected. While the separated sea clutter signal is quite well, see Fig 6. The red line is estimated signal and the green line 

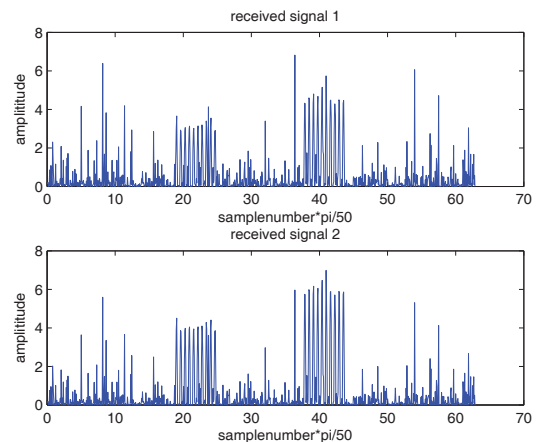

Fig. 5. Two example received signals

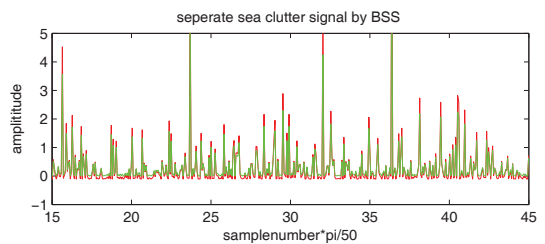

Fig. 6. Separated sea clutter signal by BSS, the red line is estimated signal and the green line is the original signal

is the original signal. So the separated sea clutter signal can be used as the reference signal of AIC.

From the results in Fig.7 and Fig.8, it is clear that the result of the proposed structure and algorithm is much better. The result of the AIC shows that it misses some targets because of its convergence. Moreover, the SNR also verifies that the proposed approach and algorithm is much better.

$$
\operatorname{SNR}(d B)=10 \log _{10}\left(\frac{P_{\text {signal }}}{P_{\text {noise }}}\right)
$$

where $P_{\text {signal }}$ is the power of signal and $P_{\text {noise }}$ is the power of noise. The SNR of $s_{1}$ is $2.04 d B$, and the SNR of AIC structure is $4.34 d B$, while the SNR of the proposed approach is $10.26 \mathrm{~dB}$.

The detection ability of this new scheme can be measured by the false alarm rate (FAR).

$$
F A R=\frac{N_{\text {false }}}{N_{\text {total }}}
$$

$N_{\text {false }}$ is the sample number of false detection, and $N_{\text {total }}$ is the total sample number. The interval between the two targets is used to do this experiment. Ideally, all the values of the sample numbers between the two targets should be zero. In practice, a threshold is set to detect the false alarm rate. If the value of a sample is greater than the threshold, it is a false alarm. In this experiment, the threshold is set as 0.1 times of the first received target value. The FAR of original signal is 0.09 , and the FAR of the de-noised signal dealt with by the proposed scheme is 0 .

\section{CONCLUSION}

In this paper, a new adaptive processing structure is proposed to solve the sea clutter problem of a radar signal in
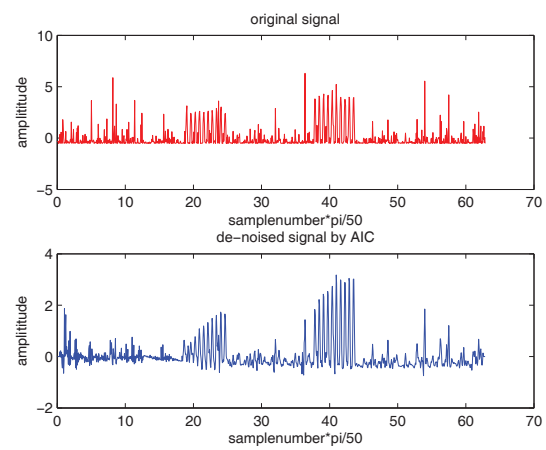

Fig. 7. Results with the AIC
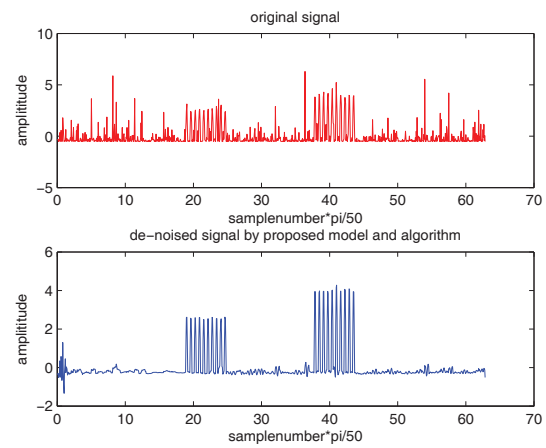

Fig. 8. Results with the proposed structure using FastICA and RLS algorithms

the navigation system. The new approach combines BSS and AIC. The separated sea clutter by BSS can be used as the reference input of the AIC. The experiment results shows that the proposed structure and algorithm is better than the AIC objectively in term of the performance index such as SNR and FAR and subjectively according to visual observation of the simulation results.

\section{REFERENCES}

[1] H. Rohling, "Radar CFAR thresholding in clutter and multiple target situations," IEEE Transactions on Aerospace and Electronic Systems, vol. AES-19, pp. 608-621, 1983.

[2] Z. Hammoudi and F. Soltani, "Distributed IVI-CFAR detection in nonhomogeneous environments," Signal Processing, vol. 84, pp. 1231-1237, 2004.

[3] J.F. Cardoso, "Blind signal separation: statistical principles," Proceedings of the IEEE, vol. 86, pp. 2009-2025, 1998.

[4] B. Widrow and J.R. Glover, "Adaptive noise cancelling: Principles and applications," Proceeding of the IEEE, vol. 63, pp. 1692-1716, 1975.

[5] D.C. Schleher, "Radar detection in Weibull clutter," IEEE Transactions on Aerospace and Electronic Systems, vol. AES-12, pp. 736-743, 1976.

[6] J. Karhunen A. Hyvarinen and E. Oja, "Independent component analysis," Wiley-Interscience, pp. 182-196, 2001.

[7] A.H. Sayed, "Adaptive filters," Wiley-Interscience, p. 198, 2008.

[8] S. Haykin, "Adaptve filter theory," Prentice-Hall, pp. 390-396, 1986. 